\title{
Portal Hypertension and Related Clinical Syndromes
}

\author{
Aly Saber ${ }^{*}$ and Tahir M. Bajwa ${ }^{2}$ \\ ${ }^{1}$ Department of general surgery, Port-Fouad General Hospital, Port-Said, Egypt \\ ${ }^{2}$ Department of general surgery, Services hospital, Lahore, Pakistan
}

Received: February 25, 2014; Accepted: June 05, 2014; Published: October 13, 2014

*Corresponding author: Aly Saber, Department of General Surgery, Port-Fouad General Hospital, Port-Said, Egypt, E-mail: Alysaber54@gmail. com

\begin{abstract}
Portal hypertension is characterized by an increased portal pressure gradient which is the difference in pressure between the portal vein and the inferior vena cava and becomes clinically significant when the portal pressure gradient increases to 10 $\mathrm{mmHg}$ or above. Portal hypertension is responsible for many of the manifestations of liver cirrhosis. These manifestations such as gastrointestinal bleeding as a result of ruptured gastroesophageal varices and portal hypertensive gastropathy, colopathy and ascites are documented. Hepatorenal syndrome and hypersplenism are the direct consequences of portal hypertension. Stellate cell activation is a key event in liver injury, and indicates the transition from a quiescent vitamin A-rich cell to a highly fibrogenic cell. Activation consists of three major phases: initiation, perpetuation and resolution. Initiation refers to early paracrine-mediated changes in gene expression and phenotype that render the cells responsive to other cytokines and stimuli while perpetuation results from the effects of these stimuli on maintaining the activated phenotype and generating fibrosis. Portal hypertension secondary to hepatic fibrosis and cirrhosis has multisystem effects and multiple complications. The multisystem effects are hepatorenal, portopulmonary hypertension, hepatopulmonary syndrome, portal hypertensive colopathy and others. Once a patient develops any of such complications, he is considered to have decompensated disease with the high morbidity and mortality.
\end{abstract}

\section{Background}

Portal hypertension is a syndrome characterized by an increased Portal Pressure Gradient (PPG) which is the difference in pressure between the portal vein and the inferior vena cava. This increase in portal pressure leads to development of an extensive network of portal-systemic collaterals that shunt portal blood flow to the systemic circulation bypassing the liver and development of a hyperkinetic circulatory state. In normal conditions the PPG ranges between 1 and $5 \mathrm{mmHg}$. Portal hypertension becomes significant from the clinical point of view when the PPG increases to $10 \mathrm{mmHg}$ or above while values between 5 and $9 \mathrm{mmHg}$ represent subclinical portal hypertension [1]. The liver with normal functions, the intrahepatic resistance changes with variations in portal blood flow to keep portal pressure within normal limits. However, in hepatic cirrhosis, the intrahepatic resistance and splanchnic blood flow are increased [2].
The portal hypertension is initiated by the increased vascular resistance to portal blood flow and is primarily caused by structural changes such as fibrotic scar tissue and regenerative nodules compressing portal and central venules. Furthermore, previous reports showed that swelling of hepatocytes and capillarisation of hepatic sinusoids (loss of endothelial fenestrations and collagen deposition in the space of Disse) are part of the increased vascular resistance. The Increase in the portal vein blood flow occurs in a more advanced stage of portal hypertension and contributes to its maintenance and aggravation [1,2]. Portal hypertension is usually caused by pre-hepatic abnormalities (e. g. portal or splenic vein thrombosis), post-hepatic abnormalities (e. g. Budd-Chiari syndrome) or intrahepatic non-cirrhotic causes (e. g. schistosomiasis, sinusoidal obstruction syndrome) however cirrhosis is by far the most common cause of portal hypertension and has been the most widely investigated [3].

Portal hypertension is responsible for many of the manifestations of liver cirrhosis. Gastrointestinal bleeding as a result of ruptured gastroesophageal varices and from portal hypertensive gastropathy and colopathy, ascites and hepatorenal syndrome and hypersplenism are the direct consequences of portal hypertension itself. Other complications including spontaneous bacterial peritonitis, hepatic encephalopathy, cirrhotic cardiomyopathy, hepatopulmonary syndrome, and portopulmonary hypertension are also caused by pathophysiological pathway of portal hypertension [4].

\section{Pathophysiology of Portal Hypertension}

Portal hypertension is primarily caused by the increase in both resistance to portal blood outflow and secondly by an increase in splanchnic blood flow and is associated with changes in the intrahepatic, systemic, and portosystemic collateral circulation. Alterations in vasodilatation and vasoconstriction play a central and pivotal role in the pathophysiology of portal hypertension by contributing to increased intrahepatic resistance, hyperdynamic circulation, and expansion of the collateral circulation [5].

\section{Hepatic vasodilators}

Nitric oxide: Nitric Oxide (NO), as a powerful endogenous vasodilator, modulates the intrahepatic vascular tone and is produced from the amino acid L-arginine by NO synthases. However, in the cirrhotic liver, NO synthesis is insufficient 
to compensate for the activation of vasoconstrictor systems frequently associated with cirrhosis. The insufficient hepatic NO production may account for the increased intrahepatic vascular resistance in cirrhosis, thereby worsening portal hypertension [6].

Carbon monoxide: As a result of heme group oxidation by heme oxygenases, Carbon Monoxide (CO) is produced and is considered as an important modulator of intrahepatic vascular resistance in spite of being less potent than NO. The inhibition of Carbon monoxide production increases portal resistance in normal livers. In addition, plasma CO levels directly correlated with cardiac output and inversely with systemic vascular resistance and mean arterial pressure. Accordingly, CO may be closely related to the hyperdynamic circulatory state in cirrhosis [7].

\section{Splanchnic vasodilatation}

Portal venous blood inflow tends to increase in cirrhosis, particularly in advanced stages of portal hypertension, due to the vasodilatation in the splanchnic organ. The increase in blood flow is one of the key factors which contribute to the pathophysiology of portal hypertension [6]. There are some possible mechanisms which account for the portal hemodynamic abnormalities as neurogenic, humoral, and local mechanisms. Vasodilators in the systemic circulation have been investigated to explain the pathophysiology of portal hypertension. The increased levels of these vasodilators are detected with impaired hepatic function or development of portosystemic collaterals because most of them are metabolized in the liver $[4,6]$.

Nitric oxide: Nitric Oxide is involved in the regulation of splanchnic and systemic hemodynamics in portal hypertension. Excessive production of NO may be one of the major reasons for the vasodilatation where splanchnic vasoconstrictive effect caused by NO inhibitors in animal studies is significantly greater in portal hypertension model than control model [6]. Furthermore, the fact that cirrhotic patients show increased levels of serum and urinary concentrations of products of NO oxidation; nitrite and nitrate, also supports a role of NO in the pathophysiology of portal hypertension $[8,9]$.

Glucagon: Glucagon is a humoral vasodilator which is associated with splanchnic hyperemia and portal hypertension. Glucagon has two mechanisms; relaxing the vascular smooth muscle and decreasing its sensitivity to endogenous vasoconstrictors. These vasoconstrictors are norepinephrine, angiotensin II, and vasopressin. Plasma glucagon levels are increased in patients with liver cirrhosis and experimental models of portal hypertension as a result of decreased hepatic clearance of glucagon as well as an increased secretion of glucagon by pancreatic $\alpha$ cells. The role of glucagon in the splanchnic hyperemia of portal hypertension provides a rationale for the use of somatostatin and its synthetic analogs to reduce glucagon level, thereby treating portal hypertension [6].

\section{Hyperdynamic circulation}

The portal hypertension is directly related to portal inflow and resistance, as determined by Ohm's law (portal pressure $=$ portal venous inflow $\times$ outflow resistance) [2]. Portal venous blood inflow is affected by hyperdynamic circulation, which is characterized by systemic and splanchnic vasodilatation, low systemic resistance, expansion of plasma volume, and higher cardiac index $[1,2]$. Splanchnic vasodilatation helps contributing to increasing substantial blood volume which returns to portal venous system. Peripheral vasodilatation activates the endogenous neurohumoral systems causing sodium retention, which leads to plasma volume to expand and therefore followed by an increase in the cardiac index. Expansion of plasma volume is a necessary step to maintain an increased cardiac index that allows in turn aggravating portal hypertension. This fact provides the rationale for using a low-sodium diet and diuretics in the treatment of portal hypertension [6].

\section{Portosystemic collateral circulation}

The development of Porto-collateral circulation is one of the hemodynamic features of portal hypertension. Formation of collaterals is a complex process involving the opening, dilatation, and hypertrophy of pre-existing porto-systemic vascular channels. Collaterals develop according to the increased portal pressure, and minimum threshold level of Hepatic Venous Pressure Gradient (HVPG) may be $10 \mathrm{mmHg}$ for the development of portosystemic collaterals and esophageal varices [10]. The vascular resistance of collateral circulation may be considered as a major component of the overall resistance to portal blood flow and, therefore, may be an important factor in determining portal pressure. In addition, the hyperdynamic splanchnic circulation state associated with portal hypertension is the consequence of active splanchnic vasodilatation; recent data suggests that the increased neovascularization in splanchnic organs plays an important role in increasing splanchnic blood inflow [6].

\section{Vasoconstrictors and hepatic vascular bed}

Endothelins: Endothelins (ETs) are a family of homologous 21 amino acid peptides which include ET-1, ET-2, ET-3, and ET-4. These endothelins exert various biological effects, vasoconstriction, and stimulation of cell proliferation in tissue and one of the major roles of endothelins is to modulate the vascular tone in cirrhosis. Plasma levels of ET-1 and ET-3 are increased in patients with liver cirrhosis and the level is dominant in patients with ascites [11].

Angiotensin II: Angiotensin II is a powerful vasoconstrictor agent, which may contribute to increasing hepatic vascular resistance. Angiotensin-II antagonists, inhibitors of the converting enzyme, or Angiotensin-II receptors blockers may have a potential to reduce portal pressure, though their effects may be accompanied with systemic hypotension $[1,2]$.

Norepinephrine: Norepinephrine is also a powerful vasoconstrictor agent, which is involved in the regulation of hepatic vascular tone. Meanwhile, the administration 
of $\alpha$-adrenergic antagonists would inhibit the increase of resistance by norepinephrine. In addition, the hepatic vascular bed of cirrhotic livers exhibits an exaggerated response to the $\alpha$-adrenergic agonist Methoxamine. This exaggerated response is associated with the over production of Thromboxane A2 (TXA2) by COX-1 isoenzyme and is completely corrected by the use of nonselective COX blockers, COX-1-selective blockers, or TXA2 antagonists. Therefore, an increased production of TXA2 markedly enhances the vasoconstrictive response of the hepatic vascular bed to methoxamine in cirrhotic patients [6].

\section{Hepatic endothelial dysfunction}

In general and under normal condition, the endothelium has a function to produce vasodilators in response to increases in blood volume and blood pressure or to produce vasoconstrictors to prevent or attenuate the concomitant increase in pressure. The abnormality in the endothelium-related vascular reaction termed as endothelial dysfunction occurs in several pathologic conditions [12]. Indeed, studies performed both in patients with cirrhotic livers and in experimental models have shown that the cirrhotic liver cannot accommodate the increased portal blood flow caused by the postprandial hyperemia, which determines an abrupt postprandial increase in portal pressure and this observation is in contrary to what happens in normal livers [6].

Endothelial dysfunction in liver cirrhosis has been attributed to the reduction in bioavailability of nitric oxide and to increased vasoconstrictors. Such imbalance between endogenous vasoconstrictor and vasodilator factors observed in the cirrhotic liver is quite similar to that found in the systemic circulation in patients with arterial hypertension. This is why the extensive advances in cardiovascular research have contributed enormously to an improved knowledge of the pathophysiology of portal hypertension. The main factors involved are described below [12].

\section{Hepatic Stellate Cells (HSCs)}

Hepatic Stellate Cells (HSCs) represent 5-8\% of all human liver cells and $13 \%$ of the volume of sinusoidal cells. Stellate cells are located in the perisinusoidal space of disse beneath the endothelial barrier and undergo a gradual transition from the quiescent stage, vitamin A storing phenotype to the activated myofibroblast-like phenotype after liver injury. Hepatic stellate cells are the main contributors to biliary liver fibrosis, to toxic liver fibrosis and are the principal source of myofibroblasts in cholestatic liver fibrosis [2]. These activated stellate cells can synthesize large amounts of extracellular matrix proteins, such as collagens I, III, IV, V, and VI, fibronectin, laminin, and proteoglycans, during liver fibrogenesis. Hepatic stellate cells have long cytoplasmic processes running parallel to the sinusoidal endothelial wall, make contact with numerous hepatocytes, and function as liver-specific pericytes. As hepatic stellate cells contract and relax in response to various vasoactive mediators, they may play a role in the regulation of sinusoidal tone and blood flow in normal liver [13].

\section{Function of HSCs}

Vitamin A storage: Hepatic stellate cells can store $80 \%$ of retinoids in the whole body as retinyl palmitate within lipid droplets in the cytoplasm. Under normal physiological conditions, these cells play pivotal roles in the regulation of retinoid homeostasis by expressing specific receptors for retinol-binding protein on their cell surface. While under pathological conditions such as hepatic fibrosis or cirrhosis, HSCs lose vitamin A and synthesize a large amount of extracellular matrix components including collagen, proteoglycan, glycosaminoglycan and adhesive glycoproteins [14].

Blood flow regulation in the normal liver: The hepatic sinusoid as the principal site of blood flow regulation is the narrowest vascular structure within the liver; and the site of highest vascular resistance. Moreover, the hepatic sinusoid is the vital site for transvascular exchange between blood and hepatocytes. The sinusoidal surface of the hepatocytes is separated from blood by many types of cells. These cells are the fenestrated sinusoidal endothelial cells that act for lining the sinusoid, Kupffer cells; liver macrophages protruding into the lumen of the sinusoid, Pit cells; liver specific natural killer cells and HSCs. There are four important potential sites for sinusoidal blood flow regulation: (i) Portal venules, (ii) Hepatic arterioles, (iii) Central venules, and (iv) Sinusoids, with inlet sphincters, outlet sphincters, and sinusoidal cells of which HSC and sinusoidal endothelial cells have contractile properties [2].

Immunoregulation: The emergence of stellate cells as significant mediators of hepatic immunoregulation has been among the most surprising discoveries about these cells. Stellate cells act to amplify the inflammatory response by inducing infiltration of monocytes and polymorphonuclear leukocytes. Stellate cells also can function as professional antigen presenting cells that can stimulate lymphocyte proliferation or apoptosis. In addition to mononuclear cell chemoattractants, stellate cells produce neutrophil chemoattractants contributing to the neutrophil accumulation characteristic of alcoholic liver disease, as well as complement protein C4 which contributes to the liver's inflammatory response [15].

Production and degradation of ECM by HSCs: In pathological conditions such as liver cirrhosis, HSCs lose vitamin A, proliferate vigorously and synthesize and secrete a large amount of ECM components. The structure of the cells also changes from starshaped HSCs to that of fibroblast-like cells or myofibroblasts. MMPs (Matrix Metalloproteinases) and TIMP (Tissue Inhibitor of Metalloproteinases) have been reported to be synthesized by HSCs. Recent reports indicate that a differential expression of MMP activity and remodeling of ECM components are dependent on the substratum used for the culture of HSCs. HSCs can take up collagen fibrils by enodocytosis and phagocytosis for degradation [14].

Production of growth factors and cytokines: Stellate cells are an important source of cytokines in the liver. They secrete TGF and Epidermal Growth Factor (EGF), two potent epithelial growth 
factors that play important roles in hepatocytes proliferation during liver regeneration. TGF and EGF also stimulate mitosis in stellate cells, creating an autocrine loop for cellular activation. Hepatocyte Growth Factor (HGF) is a more potent hepatocyte mitogen produced by stellate cells which diminishes during acute liver injury. Stem Cell Factor (SCF) has also been identified in stellate cells in rats undergoing liver regeneration induced by partial hepatectomy combined with 2-acetoaminofluorene. In addition, Insulin-like Growth Factor (IGF) I and II are secreted by stellate cells [15].

\section{Activation of HSCs}

Stellate cell activation is a key event in liver injury and refers to the transition from a quiescent vitamin A-rich cell to a highly fibrogenic cell $[15,16]$ and those cells with features of both quiescent and activated states are often called transitional cells. A recent study reported that hepatic stellate cells together with portal fibroblasts are believed as the major source of myofibroblast that participate in hepatic fibrogenesis by way of synthesis of proinflammatory cytokines and extracellular matrices [17].

Proliferation of stellate cells occurs in regions of greatest injury, and is typically preceded by an influx of inflammatory cells and associated with subsequent Extracellular Matrix (ECM) accumulation. Activation consists of three major phases: initiation, perpetuation and resolution. Initiation refers to early paracrine-mediated changes in gene expression and phenotype that render the cells responsive to other mediators; cytokines and stimuli. Perpetuation then results from the effects of these stimuli on maintaining the activated phenotype and generating fibrosis [15-17].

Initiation phase: The earliest changes in stellate cells are likely to result from paracrine stimulation by all neighboring parenchymal and non parenchymal cell types, including sinusoidal endothelium, Kupffer cells, hepatocytes, platelets, and leukocytes. Endothelial cells are also likely to participate in the process of activation, both by production of cellular fibronectin and via conversion of Transforming Growth Factor (TGF)-beta from the latent to active profibrogenic form. Kupffer cell infiltration and activation also play a prominent role in the initiation phase where influx of these cells coincides with the appearance of stellate cell activation markers. Kupffer cells can stimulate matrix synthesis, cell proliferation, and release of retinoids by stellate cells through the actions of cytokines especially, TGF-beta 1. In addition, Kupffer cells produce both anti-inflammatory as well as proinflammatory cytokines, including interleukin (IL)-10. Hepatocytes are a potent source of fibrogenic lipid peroxides in inflammatory liver diseases. Platelets are a potent source of growth factors $[18,19]$ and in liver injury potentially important platelet mediators including Platelet Derived Growth Factor (PDGF), TGF-beta 1, and Epidermal Growth Factor (EGF) are released[17].

Perpetuation phase: Phase of perpetuation of stellate cell activation involves the following changes in cell behavior: proliferation, chemotaxis, fibrogenesis, contractility, matrix degradation, retinoid loss, and cytokine release. The net effect of these changes is to increase accumulation of extracellular matrix. As an example, proliferation and chemotaxis lead to increased numbers of collagen-producing cells. Cytokine release by stellate cells can amplify the inflammatory and fibrogenic tissue responses [15].

a) Proliferation: Platelet Derived Growth Factor (PDGF) is the most identified potent stellate cell mitogen and induction of PDGF receptors early in stellate cell activation increases responsiveness to this potent mitogen. Other compounds with mitogenic activity in stellate cells and a potential role in fibrogenesis include vascular endothelial cell growth factor, thrombin and its receptor, EGF, TGF- $\alpha$, keratinocyte growth factor, and bFGF. Signaling pathways for all these mitogens have been greatly clarified in stellate cells, offering many potential sites for therapeutic intervention $[15,18]$.

b) Chemotaxis: Stellate cells have the ability to migrate towards cytokine chemoattractants and other chemoattractants such as PDGF. The mechanical features of stellate cell chemotaxis have recently been explored and revealed that PDGF-stimulated chemotaxis is associated with cell spreading at the tip, movement of the cell body towards the stimulant, and retraction of trailing protrusions associated with transient myosin phosphorylation $[15,16,18]$.

c) Fibrogenesis: Stellate cells can generate fibrosis by increasing the cell numbers and matrix production per cell. The most potent stimulus for production of collagen I and other matrix constituents by stellate cells is TGF- $\beta$, which is derived from both paracrine and autocrine sources $[15,19]$.

d) Contractility: Contractility of stellate cells may be a major determinant of early and late increases in portal resistance during liver fibrosis where impeding portal blood flow by constricting individual sinusoids and by contracting the cirrhotic liver. The acquisition of a contractile phenotype during stellate cell activation has been documented in culture and in vivo and is mediated in part by receptors that interact with the extracellular matrix and are driven by calcium signaling [15,17].

e) Matrix degradation: The process of fibrosis reflects a balance between matrix production and degradation while degradation of extracellular matrix is a key event in hepatic fibrosis and replacement by scar matrix. Stellate cells are the principal source of MMP-2, MMP-9, MMP-13, and stromelysin. Activation of latent MMP-2 may require interaction with hepatocytes. Markedly increased expression of MMP-2 is characteristic of cirrhosis $[15,17]$.

Resolution phase: Two potential pathways account for reduction in activated stellate cells, either through reversion to a quiescent phenotype or clearance through apoptosis [15]. Some of the mechanisms responsible for these two potential pathways; fibrogenesis and fibrosis regression get focusing on the role of hepatic stellate cells activation and apoptosis, the importance of matrix metalloproteinases and their tissue inhibitors and the 
central involvement of hepatic macrophages in orchestrating this process [20].

\section{HSCs and portal hypertension}

The initial event in the pathophysiology of portal hypertension is increased vascular resistance to portal flow caused by structural changes such as fibrotic scar tissue and regenerative nodules compressing portal and central venules [2].

The anatomic location of stellate cells: The anatomic location of stellate cells in the perisinusoidal space in the normal human liver plays an important role in the regulation of sinusoidal blood flow. These cells reside and extend elongate protrusions that run along and encircle one or more sinusoids. This anatomy is similar to that of tissue pericytes, such as the mesangial cells of the kidney which can modulate vascular tone by contracting around their capillaries. In the same way, hepatic stellate cells have been theorized to regulate sinusoidal resistance, and consequently blood flow, by contracting around sinusoids [22].

Increased intrahepatic resistance: HSCs play a main role in excessive production and accumulation of extracellular matrix in liver fibrosis and have been implicated in various liver disease processes other than fibrosis. Contraction of activated HSCs may have important implications in the pathogenesis of portal hypertension, in the contraction of mature scar tissue and contributing to the dysregulation of microvascular blood flow [22]. The imbalance between overproduction of vasoconstrictors and the impaired production of vasodilators are the main events of the increased vascular tone in the sinusoidal area of the liver. In addition to an imbalance in vasoactive substances which is considered a major role attributing to activated hypercontractile hepatic stellate cells which cause vascular remodeling as an adaptive response to the changed balance in vasoactive substances [23].

\section{Related Clinical Syndrome}

\section{Hepatorenal syndrome}

Hepatorenal Syndrome (HRS) is a functional renal failure that often occurs in patients with cirrhosis and ascites. There are two different types of HRS that have been described. Type 1 HRS develops as a consequence of a severe reduction of effective circulating volume due to both an extreme splanchnic arterial vasodilatation and a reduction of cardiac output while Type 2 HRS is characterized by a stable or slowly progressive renal failure [24].

Pathophysiology of hepatorenal syndrome: The main pathophysiological mechanisms of hepatorenal syndrome include increased renal arterial resistance, especially affecting the renal cortex resulting in renal hypoperfusion, and arterial hypotension. The small volume of the ultra filtrate is reabsorbed almost completely in the proximal tubules whereas almost a zero quantity of sodium flows to the loop of Henley. As a result of these mechanisms, enhanced aldosterone activity is of little effect in this phase as well as the standard diuretics. The principal mechanisms leading to renal vasoconstriction consist of alterations in systemic circulation, accompanying portal hypertension, which are represented by decreased peripheral vascular resistance with subsequent vasodilatation, central hypervolemia, hyperkinetic circulation, and the activation of compensatory mechanisms, i. e. , the Sympathetic Nervous System (SNS), Renin Angiotensin Aldosterone System (RAAS) and adiuretin-vasopressin. Arterial hypotension is the key factor which causes simultaneous renal vasoconstriction and renal hypoperfusion with decreased glomerular filtration [25].

Clinical presentation: HRS occurs almost exclusively in patients with ascites and liver cirrhosis and the prevalence of HRS in those patients is equal to $18 \%$ after 1 year reaching up to $39 \%$ at 5 years. From the pathophysiological point of view, HRS can be classified in two main types: Type 1; rapidly progressive with serum creatinine gets doubled in 2 weeks and values of 350 $\mu \mathrm{mol} / \mathrm{L}(2.5 \mathrm{mg} / \mathrm{dL})$ are usually achieved. This type accompanies the more clinically serious conditions and it is typically unstable and acute renal failure is main clinical feature. Type 2; slowly progressive signs of hepatorenal failure with serum creatinine rises slowly or not at all and it usually does not exceed $180 \mu \mathrm{mol} / \mathrm{L}$ (1. $3 \mathrm{mg} / \mathrm{dL}$ ). The clinical record is dominated by refractory ascites and relatively stable liver function [26].

Diagnosis of HRS: There is no specific test to determine the proper diagnosis of HRS. The prime finding consists of reduced glomerular filtration (creatinine clearance) $<40 \mathrm{~mL} /$ min or serum creatinine increase $>135 \mu \mathrm{mol} / \mathrm{L}$ with exclusion of other causes of renal failure. The most relevant indications of the functional character of such failure include: natriuresis $<10 \mathrm{mmol} / \mathrm{L}$, urine osmolality higher than plasma osmolality, natremia < $130 \mathrm{mmol} / \mathrm{L}$ and diuresis $<500 \mathrm{~mL} / \mathrm{d}[25,27]$.

Management of HRS: The most appropriate therapy for HRS is liver transplantation but with a high rate of mortality and survival among liver transplant recipients is lower in HRS than among their counterparts without HRS. Use of terlipressin represents a milestone in the management of HRS as it causes reversal of HRS by improving renal blood flow. However, noradrenaline is another good choice. Vasoconstrictor drugs alone or with albumin reduce mortality with beneficial results as a bridge to liver transplant. The effect of Transjugular Intrahepatic Portosystemic Shunt (TIPS) on renal function and hemodynamics has been investigated and proved to be effective even in patients with cirrhosis and parenchymal renal disease. Patients with advanced cirrhosis are not good candidates to TIPS procedure as they are at risk for worsening liver failure and/or hepatic encephalopathy. TIPS activity appears to be slow and beneficial in some patients only. Many clinical, biochemical and neuro-humoral parameters improve but do not fully reverse after TIPS insertion [28,29].

\section{Pulmonary complications of portal hypertension}

Portal hypertension may be associated with one of the two pulmonary vascular complications; portopulmonary hypertension and hepatopulmonary syndrome. Portopulmonary hypertension results from excessive 
vasoconstriction, vascular remodeling, and proliferative and thrombotic events within the pulmonary circulation leading to progressive right ventricular failure and finally death. On the other hand, abnormal intrapulmonary vascular dilations, profound hypoxemia, and a wide alveolar-arterial gradient are the hallmarks of the hepatopulmonary syndrome resulting in hypoxemia [30].

\section{Portopulmonary hypertension: Portopulmonary} Hypertension (POPH) is defined as Pulmonary Arterial Hypertension (PAH) associated with portal hypertension and the diagnostic criteria include the presence of portal hypertension, hemodynamic measurements of mean pulmonary artery pressure $>25 \mathrm{mmHg}$ at rest, mean pulmonary capillary wedge pressure < $15 \mathrm{mmHg}$, and pulmonary vascular resistance > 240 [30].

a) Pathophysiology of POPH: Pulmonary vascular changes include the wall and lumen of pulmonary vessels. These changes are intimal fibrosis, hypertrophy of the smooth muscle cells and fibroblasts, thrombosis, and plexiform lesions resulting from intraluminal endothelialisation or micro-aneurysms within pulmonary arterioles. In chronic liver disease, the increased blood flow due to high cardiac output causes pulmonary vascular wall shear stress with the dysregulation of numerous vasoactive, proliferative and angiogenic mediators eventually leading to the characteristic arteriopathic changes [31]. The portosystemic shunts and decreased phagocytic capacity of the liver allow circulating bacteria and bacterial endotoxins from the gastrointestinal tract to enter the pulmonary circulation. In liver cirrhosis it is suggested that the recruitment of interstitial macrophages might also contribute to the development of POPH. Furthermore, the presence of portosystemic shunts may allow the shunting of vasoactive substances from the splanchnic to the pulmonary circulation and this causes deleterious effects in the pulmonary vasculature [30,31].

b) Clinical manifestations: A high index of suspicion should be present when evaluating patients with liver disease, since POPH itself typically produces no symptoms or only has symptoms related to the underlying cirrhosis or portal hypertension. Dyspnoea on exertion is the most common presenting complaint but it is frequently related to other conditions such as refractory ascites with mechanical thoracic impairment, hepatic hydrothorax, anaemia and sarcopenia/deconditioning. Other common symptoms including fatigue, generalized weakness, light-headedness and orthopnoea are also present. In advanced stages of POPH, symptoms like oppressive chest discomfort, dyspnoea while at rest, syncopal attacks and haemoptysis can occur [32]. Physical examination may reveal elevated jugular venous pressure, an accentuated pulmonic component (P2) of the second heart sound, a holosystolic murmur consistent either with tricuspid regurgitation or pulmonic insufficiency (GrahamSteele murmur), as well as a right ventricular heave with signs of right heart failure (third or fourth heart sounds). A pulsatile liver during abdominal examination and lower extremity pitting edema out of proportion to ascites may also signal right ventricular dysfunction. Physical stigmata of cirrhosis and portal hypertension, such as splenomegaly, ascites, spider angiomata, scleral icterus, jaundice, palmar erythema, gynecomastia and asterixis may also be observed [30].

c) Diagnosis of POPH: The single most important test to screen for POPH is the two-dimensional Transthoracic Echocardiogram (TTE) which may help to show pulmonic insufficiency, right ventricular hypertrophy, dilatation and dysfunction, as well as right atrial enlargement that could be indirect signs of significant Pulmonary Arterial Hypertension (PAH). Paradoxical septal movement is also common [32].

d) Management of POPH: The general goals of therapy for POPH are to provide symptomatic relief, improve quality of life and exercise capacity, and to facilitate successful liver transplantation. The use of calcium channel blockers in POPH is generally contraindicated as they can produce mesenteric vasodilatation that can worsen portal hypertension. Despite the strong evidence and wide use of $\beta$-blockers to aid in primary and secondary prophylaxis for variceal gastrointestinal bleeding in the setting of portal hypertension, it has been demonstrated that in POPH their use has been associated with deterioration of exercise capacity and pulmonary haemodynamics, due to their negative inotropic and chronotropic effects. Oral anticoagulation is regularly recommended for many forms of Pulmonary Arterial Hypertension (PAH), but with increased risk of gastrointestinal haemorrhage. Diuretics such as furosemide and spironolactone may offer symptomatic relief in patients with right heart failure due to $\mathrm{PAH}$ and liver cirrhosis with beneficial role in $\mathrm{POPH}$ patients with volume overload and fluid retention. Close monitoring is required, however, since diuretics can reduce cardiac output by decreasing right ventricular preload, facilitating renal failure and systemic hypoperfusion [30].

Hepatopulmonary syndrome: Hepatopulmonary Syndrome (HPS) is characterized by the triad of arterial deoxygenation, intrapulmonary vascular dilatation and liver disease. HPS can occur with any degree of liver disease, ranging from well-compensated chronic liver disease without cirrhosis to noncirrhotic portal hypertension and cirrhosis and also been described in patients with acute liver failure [30].

a) Pathophysiology of HPS: The hallmark of HPS is intrapulmonary vasodilatation markedly at both the pre-capillary and capillary level of the pulmonary circulation especially in the lower lobes. The consequence of the intrapulmonary vasodilatation is arterial deoxygenation by three mechanisms: ventilation/perfusion mismatch, intrapulmonary shunting, and limitation of oxygen diffusion and these three mechanisms contribute to passing of mixed venous blood to the systemic circulation. The etiology of intrapulmonary vascular dilatations is related to an increase in pulmonary NO as a result of expression of both endothelial and inducible NO synthase (eNOS and iNOS, respectively). The link between portal hypertension and increased NO is incompletely understood but seems to be related to an increase in hepatic production of the vasoconstrictor ET-1. A local increase in pro-inflammatory mediators and 
pulmonary intravascular sequestration of macrophages leading to an increase in iNOS activity and NO production has also been described [30].

b) Clinical manifestations: The characteristic features in patients with HPS are dyspnoea, digital clubbing, and cyanosis and spider angiomas. Dyspnoea on exertion in case of HPS is probably confounded by physical deconditioning and fatigue, which are very common among patients with cirrhosis. Important manifestations such as platypnoea and orthodeoxia, the increase in dyspnoea or deoxygenation while in the standing position, are classically described in HPS. However, these are not pathognomonic features and actually orthopnoea seems to be a more frequent manifestation in HPS. As in POPH, other physical stigmata of cirrhosis and portal hypertension can be seen in patients with HPS [34].

c) Diagnosis of HPS: Chest radiographs are usually normal in patients with HPS but can manifest an increased interstitial pattern in the bases, an effect usually caused by arteriovenous shunts in severe HPS. High-resolution computed tomography can identify dilatation of the peripheral pulmonary vessels in the lung bases at early stages of HPS. Measurement of ABGs is the gold standard for identifying arterial deoxygenation and classifying HPS according to the degree of hypoxaemia and $\mathrm{PaO} 2$ is the main prognostic determinant for HPS. Contrast-Enhanced TTE (CETTE) has become the most commonly used test for identifying the intravascular vasodilatation of HPS and lung perfusion scanning with Macro-Aggregated Albumin (MAA scan) provides a quantitative assessment of the severity of the intrapulmonary vasodilatation [30].

d) Management of HPS: Currently, there are no effective medical therapies for HPS and liver transplantation is the only definitive therapy with the resultant improvement of arterial deoxygenation and intrapulmonary dilatation occurring almost universally post-transplant. Several studies have addressed the therapeutic potential of compounds affecting the mechanisms involved in the pathogenesis of HPS. Overall, results have not been promising. Supplemental oxygen remains the only proven effective therapy and it should be administered when the $\mathrm{PaO}_{2}$ reaches $<60 \mathrm{mmHg}$. Transjugular intrahepatic portosystemic shunt should not be considered a therapeutic option for HPS [28]. Several attempts have been made to inhibit the development of HPS by administering nitric oxide, using diets low in L-arginine using methylene blue, which is an inhibitor of guanylate cyclase, aspirin, somatostatin, almitrine, $\mathrm{N}$-acetylcysteine, indomethacin, garlic, mycophenolate mofetil (an inhibitor of angiogenesis and nitric oxide production), pentoxifylline, and using antibiotics to decrease bacterial translocation in the bowel. However, a role for any of these drugs in the long-term treatment of HPS has not been demonstrated [35].

Portal hypertensive colopathy: Portal Hypertensive Colopathy (PHC) is a frequent complication of chronic liver disease and it may cause lower gastrointestinal bleeding or unidentified chronic anemia in patients with severe portal hypertension [36]. a) Endoscopic findings: Endoscopic findings in colons of patients with portal hypertension are studied regarding the mucosal and vascular changes. The diagnosed portal hypertensive vascular colopathy are usually classified into four types; solitary and diffuse vascular ectasias, redness in the whole colon and blue rectal vein. Solitary vascular ectasias are found predominantly in the transverse and ascending colon, diffuse vascular ectasias are found predominantly in the right side colon, redness is found in the overall colon and blue vein in the rectum [37]. Mucosal abnormalities in portal hypertension colopathy include edema, erythema, granularity, friability, and vascular lesions, findings that may be confused with colitis [38].

b) Histopathological study:Dilated tortuous mucosal capillaries with irregular thickening of wall, edema of lamina propria and mild chronic inflammatory infiltrate are the major histopathological changes seen in colonic biopsies of patients with portal hypertension, showing that Portal Hypertension (PHT) produces changes in the colonic mucosa similar to those seen in the mucosa of upper GI tract [39].

c) Clinical manifestations:Portal hypertensive colopathy may be confused with other causes of colitis and while this disease entity rarely causes bleeding [4]. The lesions of portal hypertensive colopathy are more frequently present in patients with more severe esophageal varices and thrombocytopenia [40]. Child-Pugh class B and C are significantly associated with Portal hypertensive colopathy. Portal hypertensive gastropathy, esophageal varices, ascites and hepatocellular carcinoma are not related to occurrence of portal hypertensive colopathy. Platelet count was significantly decreased in association with portal hypertensive colopathy, but prothrombin time, serum albumin level, total bilirubin level and serum Alanine Transaminase (ALT) level are not usually related to occurrence of portal hypertensive colopathy [37].

d) Management of PHC:Vasoactive medications, such as octreotide or terlipressin, could be effective in patients with acute bleeding and nonselective $\beta$-blockers are recommended as soon as hemodynamic stability is achieved. The use of neodymium:yttrium-aluminum-garnet laser photocoagulation therapy to remove the angioectasias with the probability of remaining free of bleeding for a relatively long period. The portosystemic gradient was reduced after TIPS placement with evidence of reduction in the size and number of colonic lesions. The patient was followed up for 18 months without recurrence of GI bleeding. A better control of lower GI bleeding from numerous angiodysplastic spots in the right colon was achieved after a proximal splenorenal shunt with splenectomy [41]. Colonic varices may be treated with sclerotherapy, surgical ligation and cryosurgery for treatment of anorectal varices. With lower GI bleeding refractory to medical therapy, endoscopic treatment, and TIPS, surgery may be considered [42].

\section{Conclusion}

Portal hypertension secondary to hepatic fibrosis and cirrhosis has multisystem effects and multiple complications. 
The multisystem effects are hepatorenal, portopulmonary hypertension, hepatopulmonary syndrome, portal hypertensive colopathy and others. Once a patient develops any of such complications, he is considered to have decompensated disease with the high morbidity and mortality. Both the quality of life and survival of patients with cirrhosis can be improved by both prevention and treatment of these complications.

\section{References}

1. Berzigotti A, Seijo S, Reverter E, Bosch J. Assessing portal hypertension in liver diseases. Expert Rev Gastroenterol Hepatol. 2013; 7(2): 141155. doi: 10.1586/egh.12.83.

2. Mederacke I, Hsu CC, Troeger JS, Huebener P, Mu X, Dapito DH, et al. Fate tracing reveals hepatic stellate cells as dominant contributors to liver fibrosis independent of its aetiology. Nat Commun. 2013; 4 2823. doi: $10.1038 /$ ncomms3823.

3. Bari K,Garcia-Tsao G. Treatment of portal hypertension. World J Gastroenterol. 2012; 18(11): 1166-1175. doi: 10.3748/wjg.v18. i11.1166

4. Al-Busafi SA, McNabb-Baltar J, Farag A, Hilzenrat N Clinical manifestations of portal hypertension. Int J Hepatol. 2012.

5. Cichoz-Lach H, Celinski K, Slomka M, Kasztelan-Szczerbinska B Pathophysiology of portal hypertension. J Physiol Pharmacol. 2008, 59 Suppl 2: 231-238.

6. Maruyama H, Yokosuka O. Pathophysiology of portal hypertension and esophageal varices. Int J Hepatol. 2012; doi: 10.1155/2012/895787

7. Tarquini R, Masini E, La Villa G, Barletta G, Novelli M, Mastroianni R, et al. Increased plasma carbon monoxide in patients with viral cirrhosis and hyperdynamic circulation. Am J Gastroenterol. 2009; 104(4): 891 897. doi: 10.1038/ajg.2009.2.

8. Curgunlu A, Vural P, Canbaz M, Erten N, Karan MA, Tascioglu C. Plasma nitrate/nitrite and endothelin-1 in patients with liver cirrhosis. J Clin Lab Anal. 2005; 19(5): 177-81.

9. Abraldes JG, Iwakiri Y, Loureiro-Silva M, Haq O, Sessa WC, Groszmann RJ. Mild increases in portal pressure upregulate vascular endothelial growth factor and endothelial nitric oxide synthase in the intestinal microcirculatory bed, leading to a hyperdynamic state. Am J Physio Gastrointest Liver Physiol. 2006; 290(5): G980-987.

10. Kim MY, Baik SK, Lee SS. Hemodynamic alterations in cirrhosis and portal hypertension. Korean J Hepatol. 2010; 16(4): 347-352. doi: 10.3350/kjhep.2010.16.4.347

11. Cavasin MA, Semus H, Pitts K, Peng Y, Sandoval J, Chapo J, et al. Acute effects of endothelin receptor antagonists on hepatic hemodynamics of cirrhotic and noncirrhotic rats. Can J Physiol Pharmacol. 2010; 88(6): 636-43. doi: 10.1139/Y10-038.

12. Bosch J, Abraldes JG, Fernandez M, Garcia-Pagin JC. Hepatic endothelial dysfunction and abnormal angiogenesis: new targets in the treatment of portal hypertension. J Hepatol. 2010; 53(3): 558-567. doi: 10.1016/j.jhep.2010.03.021.

13. Miyata E, Masuya M, Yoshida S, Nakamura S, Kato K, Sugimoto Y, et al. Hematopoietic origin of hepatic stellate cells in the adult liver. Blood. 2008; 111(4): 2427-2435.

14. Senoo H, Yoshikawa K, Morii M, Miura M, Imai K, Mezaki Y. Hepatic stellate cell (vitamin A-storing cell) and its relative--past, present and future. Cell Biol Int. 2010; 34(12): 1247-72. doi: 10.1042/ CBI20100321.

15. Friedman SL. Hepatic Stellate Cells: Protean, Multifunctional, and Enigmatic Cells of the Liver. Physiol Rev. 2008; 88(1):125-172. doi: 10.1152/physrev.00013.2007.

16. Carpino G, Renzi A, Onori P, Gaudio E. Role of hepatic progenitor cells in nonalcoholic Fatty liver disease development: cellular cross-talks and molecular networks. Int J Mol Sci. 2013; 14(10): 20112-20130. doi: 10.3390/ijms141020112.

17. Lua I, James D, Wang J, Wang KS, Asahina K. Mesodermal mesenchymal cells give rise to myofibroblasts, but not epithelial cells, in mouse liver injury. Hepatology. 2014; 60(1):311-322. doi: 10.1002/hep.27035.

18. Melton AC, Yee HF. Hepatic stellate cell protrusions couple plateletderived growth factor-BB to chemotaxis. Hepatology. 2007; 45(6): 1446-1453.

19. Tsukada S, Parsons CJ, Rippe RA. Mechanisms of liver fibrosis. Clin Chim Acta. 2006; 364(1-2): 33-60

20. Ramachandran P, Iredale JP. Liver fibrosis: a bidirectional model of fibrogenesis and resolution. QJM. 2012; 105(9): 813-817. doi: 10.1093/qjmed/hcs069.

21. Soon RK Jr, Yee HF Jr. Stellate Cell Contraction: Role, Regulation, and Potential Therapeutic Target. Clin Liver Dis. 2008; 12(4): 791-803. doi: 10.1016/j.cld.2008.07.004.

22. Atzori L, Poli G, Perra A. Hepatic stellate cell: a star cell in the liver. Int J Biochem Cell Biol. 2009; 41(8-9): 1639-1642. doi: 10.1016/j. biocel.2009.03.001.

23. Laleman W. Role of vasoactive substances and cellular effectors in the pathophysiology of cirrhotic portal hypertension: the past, the present and the future--Georges Brohée Lecture. Acta Gastroenterol Belg. 2009; 72(1): 9-16.

24. Angeli P, Morando F, Cavallin M, Piano S. Hepatorenal syndrome. Contrib Nephrol. 2011; 174: 46-55. doi: 10.1159/000329235.

25. Lata J. Hepatorenal syndrome. World J Gastroenterol. 2012; 18(36): 4978-4984. doi: 10.3748/wjg.v18.i36.4978.

26. Gines P, Schrier RW. Renal failure in cirrhosis. N Engl J Med. 2009; 361(13): 1279-1290. doi: 10.1056/NEJMra0809139.

27. Nadim MK, Kellum JA, Davenport A, Wong F, Davis C, Pannu N,et al. Hepatorenal syndrome: the 8th international consensus conference of the Acute Dialysis Quality Initiative (ADQI) Group. Crit Care. 2012; 16(1):R23. doi: 10.1186/cc11188.

28. Fabrizi F, Aghemo A, Messa P. Hepatorenal syndrome and novel advances in its management. Kidney Blood Press Res. 2013; 37(6): 588-601. doi: 10.1159/000355739.

29. Moller S, Krag A, Bendtsen F. Kidney injury in cirrhosis: pathophysiological and therapeutic aspects of hepatorenal syndromes. Liver Int. 2014; 34(8): 1153-1163. doi: 10.1111/liv.12549.

30.Porres-Aguilar M, Gallegos-Orozco JF, Garcia H, Aguirre J, Macias-Rodriguez RU, Torre-Delgadillo A. Pulmonary vascular complications in portal hypertension and liver disease: a concise review. Rev Gastroenterol Mex. 2013;78(1):35-44. doi: 10.1016/j. rgmx.2012.10.004.

31. Farber HW, Loscalzo J. Pulmonary arterial hypertension. N Engl J Med. 2004; 351(16): 1655-1665. 
32. Huffmyer JL, Nemergut EC. Respiratory dysfunction and pulmonary disease in cirrhosis and other hepatic disorders. Respir Care. 2007; 52(8): 1030-1036.

33. Galie N, Hoeper MM, Humbert M, Torbicki A, Vachiery JL, Barbera JA, et al. Guidelines for the diagnosis and treatment of pulmonary hypertension: the Task Force for the Diagnosis and Treatment of Pulmonary Hypertension of the European Society of Cardiology (ESC) and the European Respiratory Society (ERS), endorsed by the International Society of Heart and Lung Transplantation (ISHLT). Eur Heart J. 2009; 30: 2493-2537. DOI: http://dx.doi.org/10.1093/ eurheartj/ehp297.

34. Fallon MB, Krowka MJ, Brown RS, Trotter JF, Zacks S, Roberts KE, et al. Impact of hepatopulmonary syndrome on quality of life and survival in liver transplant candidates. Gastroenterology. 2008; 135(4): 11681175. doi: 10.1053/j.gastro.2008.06.038.

35. Tumgor G. Cirrhosis and hepatopulmonary syndrome. World J Gastroenterol. 2014; 20(10): 2586-94. doi: 10.3748/wjg.v20. i10.2586.

36. Miranda MA, Ferraz AA, Domingues AL, Chaves RC, Juca N, da Mota DL. Improvement of schistosomal portal hypertensive colopathy after surgical treatment. Arq Gastroenterol. 2013; 50(2): 153-156.
37. Ito K, Shiraki K, Sakai T, Yoshimura H, Nakano T. Portal hypertensive colopathy in patients with liver cirrhosis. World J Gastroenterol. 2005; 11(20): 3127-30.

38. Bini EJ, Lascarides CE, Micale PL, Weinshel EH. Mucosal abnormalities of the colon in patients with portal hypertension: an endoscopic study. Gastrointest Endosc. 2000; 52(4): 511-6.

39. Misra V, Misra SP, Dwivedi M, Singh PA, Kumar V. Colonic mucosa in patients with portal hypertension. J Gastroenterol Hepatol. 2003; 18(3): 302-8.

40. Jeong IB, Lee TH, Lim SM, Ryu KH, Kim YS, Kim SM, et al. Endoscopic findings and clinical significance of portal hypertensive colopathy. Korean J Gastroenterol. 2011; 58(6): 332-337.

41.Urrunaga NH, Rockey DC. Portal hypertensive gastropathy and colopathy. Clin Liver Dis. 2014; 18(2): 389-406. doi: 10.1016/j. cld.2014.01.008.

42. Ozgediz D, Devine P, Garcia-Aguilar J, Balibrea JM. Refractory lower gastrointestinal bleeding from portal hypertensive colopathy. J Am Coll Surg. 2008; 207(4): 613. doi: 10.1016/j.jamcollsurg.2008.02.040. 